\title{
Effects of Humanness of Virtual Agents on Impression Formation
}

\author{
Eva Krumhuber;, Arvid Kappas \\ Jacobs University Bremen
}

\author{
Mark Hall, John Hodgson \\ University of Sunderland
}
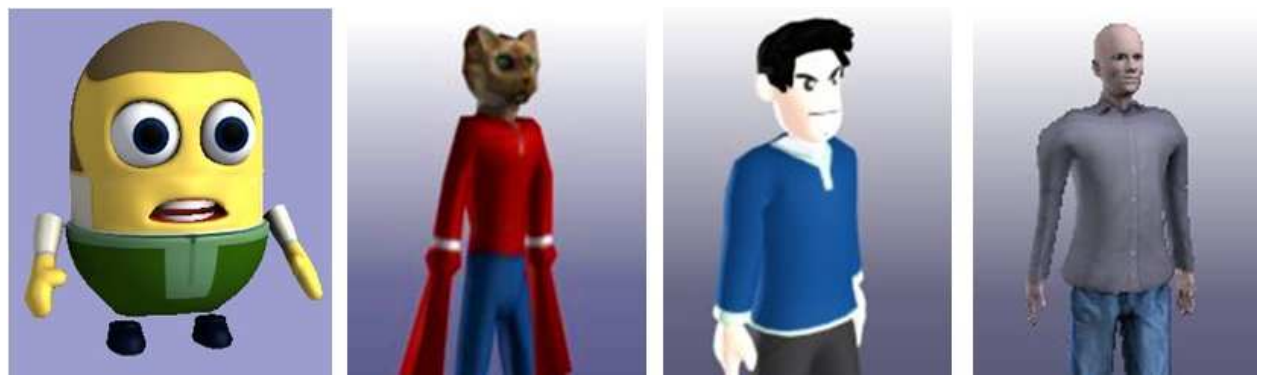

Figure 1: Four embodied characters - blob, cat, cartoon, human - from non-human to very human in a neutral position

\section{Motivation}

In recent years, the use of virtual agents that act as an interface between human and computer has become increasingly popular. Such agents typically appear as embodied characters and display various types of life-like behaviour. To ensure the acceptance with the user, questions relating to the humanness of their appearance play an important role. How realistic and human-like should a character look like? Agents which lack human realism may be less anthropomorphized and evoke fewer social responses. On the other hand, if they resemble humans too closely they may be perceived as unpleasant and produce feelings of uncanniness [Mori 1970].

\section{Our Approach}

In this paper, we argue that the acceptance of virtual agents may depend on their function or the domain in which they are used. Specifically, for entertainment purposes or ratings of enjoyment a simple interface in the form of a robotic-looking agent may be sufficient. In comparison, for educational training tasks that require the user to empathise and attribute complex mental states, agents that resemble humans are desirable. In the following, four embodied characters were created that differed in their human appearance from non-human (blob) to very human (see Figure 1). All displayed face, hands and feet and were animated to show various behaviour (idle, bow, wave). To test for a differentiated nature in perception, attributes were chosen that targeted dispositional traits such as likeability and trustworthiness, the assignment of mental abilities as well as basic and social emotions. In addition to different physical appearances, we were interested in the impression that images of the agents make in comparison to dynamic representations.

\section{Evaluation}

Forty participants aged between 18-35 years were presented with either static or dynamic displays of four embodied characters: blob, cat, cartoon, and human. In the static condition, images of the characters in a neutral position were shown for $5 \mathrm{~s}$. In the dynamic condition, each character consecutively displayed three types of movement - idle, bow, wave - which lasted about 10s. Following each stimulus presentation, participants answered on 7-point scales the following questions: (a) How likeable is the character? (b) How trustworthy is the character? (c) How intelligent is the character? (d) How engaging is the character? (e) To what degree does the character have a mind on its own? (f) To what degree can the character experience anger? (g) To what degree can the character experience shame?

Results showed that perceptions of the four embodied characters differed depending on the type of question. These effects were largely consistent across the dynamic and static condition. Overall, the blob was well liked, but ratings of intelligence and mind were lowest for this type of character. Given that it was the most non-human and object-like looking, it seems plausible that participants ascribed less mental capacities which are usually reserved for humans [Haslam et al. 2008]. This is also reflected by the finding that the cat as a living, but non-human being was seen to possess less mind than the human character. In comparison, ratings of basic (anger) and social (shame) emotions were highest for the human and cartoon character, indicating that participants perceived them as being most capable to experience complex emotional and mental states.

Together, the findings suggest that the acceptance and thus usability of embodied agents may vary with the dimension on which they are evaluated. A simple character (i.e., blob) may be sufficiently entertaining and likeable, whereas for the attribution of higher-order states agents should be closer to humans and incorporate humanlike features. The design of agents is not just an issue of realism but requires consideration of purpose-related psychological processes in users.

Acknowledgements This work has been conducted within the European Commission project eCUTE - Education in Cultural Understanding, Technologically-Enhanced (FP7-ICT-2009.4.2). We thank Tony Manstead for his help with data collection.

\section{References}

Haslam, N., Loughnan, S., Kashima, Y., and Bain, P. 2008. Attributing and denying humanness to others. European Review of Social Psychology 19, 55-85.

MORI, M. 1970. The uncanny valley. Energy 7, 33-35.

\footnotetext{
*e-mail: e.krumhuber@jacobs-university.de
} 